\title{
Preparation of Au Nanocolloids by in Situ Dispersion and Their Applications in SERS Films
}

\author{
Le Du, ${ }^{* \dagger}$ Yujun Wang, ${ }^{\star}$ Zhongqi Ren, ${ }^{*}{ }^{\dagger}$ Chun Shen, ${ }^{\S}$ and Guangsheng Luo ${ }^{\star}$ \\ ${ }^{\dagger}$ The State Key Laboratory of Chemical Resource Engineering, Beijing Key Laboratory of Membrane Science and \\ Technology, Beijing University of Chemical Technology, Beijing 100029, China \\ ${ }^{\ddagger}$ The State Key Laboratory of Chemical Engineering, Department of Chemical Engineering, Tsinghua University, \\ Beijing 100084, China \\ ${ }^{\S}$ Beijing Key Laboratory of Bioprocess, College of Life Science and Technology, Beijing University of Chemical \\ Technology, Beijing 100029, China \\ *Corresponding authors. E-mail: renzq@mai.buct.edu.cn (Zhongqi Ren), dule@mail.buct.edu.cn (Le Du)
}




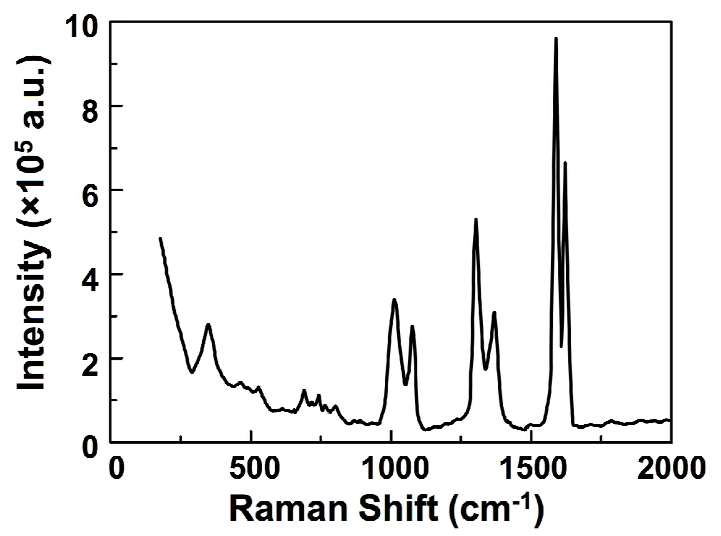

Figure S1. Normal Raman spectrum of pure BPE on the uncoated PDMS film.

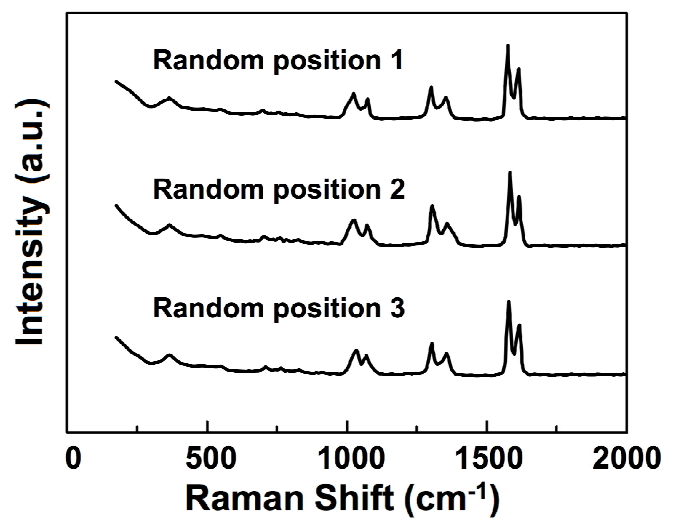

Figure S2. Raman spectra of BPE solutions across the surface of Film 1 (random positions): $100 \mathrm{ppb}$ of BPE. 


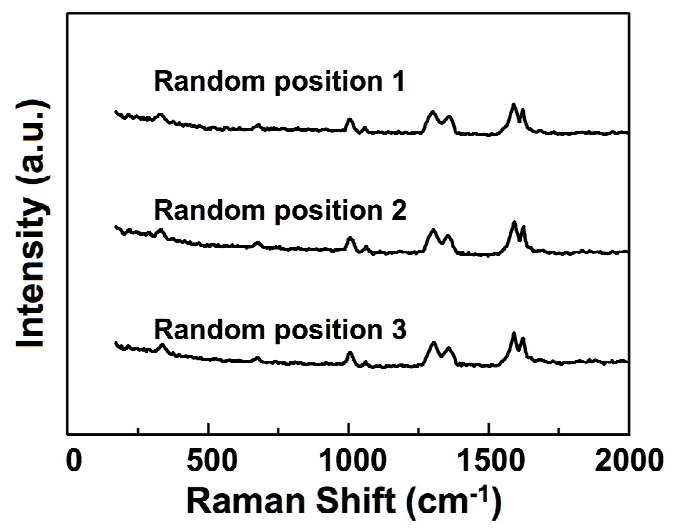

Figure S3. Raman spectra of BPE solutions across the surface of Film 1 (random positions): $10 \mathrm{ppb}$ of BPE.

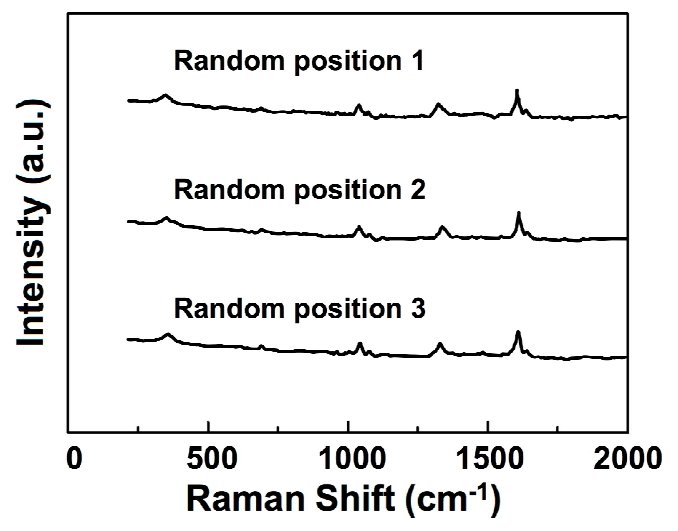

Figure S4. Raman spectra of BPE solutions across the surface of Film 2 (random positions): $100 \mathrm{ppb}$ of BPE. 


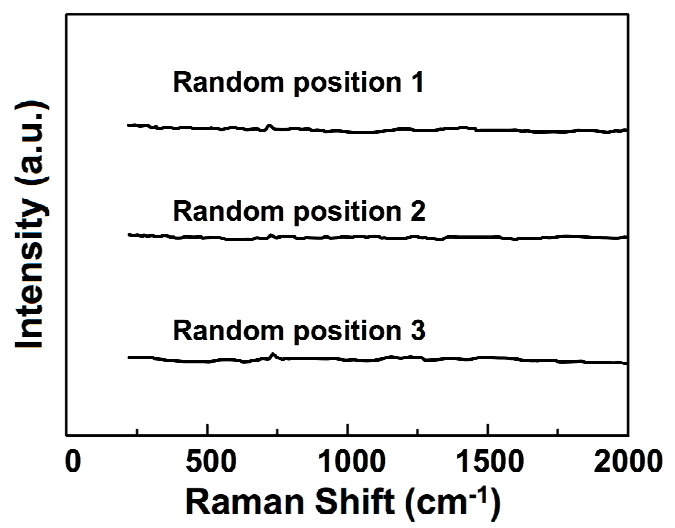

Figure S5. Raman spectra of BPE solutions across the surface of Film 2 (random positions): $10 \mathrm{ppb}$ of BPE. 